\title{
New records of butterflies and their species diversity in four different areas of Savar, Dhaka, Bangladesh
}

\author{
A T M F Islam ${ }^{1}$, M H Islam ${ }^{1}$, A S M Saifullah ${ }^{1}, \mathrm{~K} \mathrm{Endo}^{2}$ and Akira Yamanaka ${ }^{2}$ \\ ${ }^{1}$ Institute of Food and Radiation Biology, Atomic Energy research Establishment, GPO Box-3787, Dhaka-1000, Bangladesh \\ ${ }^{2}$ Department of Physics, Biology and Informatics, Faculty of Science, Yamaguchi University, Yamaguchi 753, Japan
}

\begin{abstract}
Field studies of butterflies were carried out in four different selected areas i.e. Krishnopur, AERE campus, Rajalak Farm and Horters (Part of JU campus) at Savar, Dhaka. Butterflies identified of those areas constituted 158 species belonging 10 families, 87 species of which are newly-recorded in Bangladesh. Numbers of species identified in each family reached 47 in Lycaenidae (30 newly recorded), 32 in Hesperiidae (22 new), 22 in Pieridae (11 new), 19 in Nymphalidae (6 new), 14 in Papilionidae (7 new), 14 in Saytridae (8 new), 5 in Danaidae (1 new), 3 in Riodinidae (1 new), 1 in Acraeidae and 1 newly recorded Amathusiidae. The maximum 130 species and the minimum 65 species were recorded in Krishnopur and Rajalak Farm, respectively. Almost all butterfly families were found to be dominant in Krishnopur. Among the total identified species, $35.44 \%$ belonged to very rare (VR) and $20.25 \%$ to rare (R) categories, whereas $12.18 \%, 12.03 \%$ and $13.29 \%$ species were very common (VC), common (C) and not rare (NR) categories, respectively. It may be that the numbers of butterfly species as well as their fauna vary greatly depending on the floral and ecological conditions in and around the study areas.
\end{abstract}

Key words: New record, species, diversity, butterfly, Savar, Dhaka.

\section{Introduction}

Butterflies, estimated to reach 28,000 species in the world (Robbins \& Opler, 1997), are found to inhabit various environmental conditions existing in hot to cold, moist to dry and low to high-mountain areas. Butterfly species are abundant in tropical areas, especially in tropical rainforests. Large numbers of butterfly species are identified in tropical countries including rain forests and low to high-mountain areas, for example, over 3700 species are identified in Peru. Butterfly species are reported to reach 643 in Nepal, 1163 in India, 242 in Sri Lanka and 237 in Japan. In Bangladesh, few works are available on butterfly species diversity, status and distribution except for the preliminary field studies of butterflies in Jahangirnagar University (JU) campus to clarify the fauna and its seasonal fluctuations of butterflies in Savar (Islam et al., 2007; Shahjahan et al., 2008). Savar is known as one of industrial areas of Bangladesh, but there are wide cultivating and forest areas in addition to many campuses of Government Organizations, such as Public Administration Training Centre (PATC), Bangladesh Livestock Research Institute (BLRI), Jatiya Sriti Shoudho, Centre for the Rehabilitation of the Paralyzed (CRP), Radio Station, (JU), Atomic Energy Research Establishment (AERE) and Bangladesh Krira Shikkha Prothistan (BKSP). The cultivating and forest areas as well as the campuses of those Government Organizations are covered with various vegetations and undisturbed areas for butterfly, on which various butterfly populations, suppose to be retained.

In the present study, we tried to examine the diversity, dynamics, ecological status and migration of butterfly populations in Savar, Dhaka, Bangladesh, which are changing with seasonal and habitat conditions of the area.

\section{Materials and Methods}

Selection of study areas: We selected study areas at Savar, the north-west suburbs of Dhaka City that vary in its vegetation characteristics. The meteorological conditions include a mean annual temperature of $30.5^{\circ} \mathrm{C}$ and a mean monthly rainfall of $215 \mathrm{~mm}$. We selected the following four study areas (Area-1 to Area-4) in and around Savar.

Area-1: Krishnopur, located at about $4 \mathrm{~km}$ eastsides from Savar, spreads over 200 ha, in which there is a wide habitat range. This area, low disturbed by the human interference, is composed of mixed vegetation lands cultivating for fruit plants, timber plants, grasses, flowers and vegetables. Natural vegetation including small natural forests was mainly covered with weeds and bamboo bushes.

Area-2: AERE Campus, located at about $15 \mathrm{~km}$ north of Savar and beside the Dhaka-Jumuna bridge high way, spreads over 135 ha. Vegetation includes mixed type such as fruit plant, timber plant and flower gardens, grasses in addition to building areas for offices, residences and ponds. It is a low human disturbed area. 
Area-3: Horters (Part of JU Campus), situated at about $3 \mathrm{~km}$ north of Savar and by the DhakaAricha road, spreads about 4 ha. Vegetations, mainly seedlings of fruit and flowering plants, are of mixed types. It is a high human disturbed area.

Area-4: Rajalak Farm, situated at west side of the Savar bus-stand, spreads about 9 ha. Vegetations mostly consist of fruit and herb plants in addition to some areas cultivating for shrubs and vegetables. It is also a high human disturbed area.

Identification of species: Butterflies were collected in each study area with handheld aerial sweep nets and killed immediately by pressing their thorax carefully for minimizing damages of the external organs (scales, legs and wing veins). They were identified using the specimen books "Butterflies in Thailand, Vol. 1-6 (Kurani \& Pinratana, 1981, 1983, 1985; Pinratana \& Eliot, 1982, 1996a, 1996b) and common English names (Wynter-Blyth, 1957).

Survey of the butterfly fauna: The butterflies were collected on sunny days of every month for three consecutive years from 2008 to 2010. Total numbers of days (or hours) shared for the field observations and collections of butterflies reached 144 days (ca. $576 \mathrm{hrs}$ ). Field observations and collections were made in between $10 \mathrm{am}$ and 2 $\mathrm{pm}$, when the butterflies used to be sunning in cool and cold seasons. The collected specimens were classified into one of the five categories as: very common (VC), common (C), not rare (NR), rare $(R)$ and very rare (VR) following Tiple et al. (2006, 2007).

\section{Results and Results}

One hundred fifty eight species of butterflies belonging to 10 families were identified in the present study. The number of butterfly species reached 130 in Krishnopur, 87 in AERE campus, 72 in Horters and 65 in Rajalak Farm (Table 1), of which 87 were newly recorded in Bangladesh. The rest 71 species of butterflies were identified and reported in a previous study (Islam et al., 2007).

The most dominant family of butterflies in the study areas was Lycaenidae (47 species) followed by Hesperiidae (32 species), Pieridae (22 species) and Nymphalidae (19 species). Thirty species of Lycaenid butterflies were newly recorded in Bangladesh (designated with * marks in Table 1). However, the lycaenid butterflies were found to vary among the study areas owing mostly to the differences in vegetations. All of the dominant families were found in Krishnopur. We identified 32 species of Hesperiidae (22 newly recorded) and 22 species of Pieridae (11 newly recorded). Amathusiid and riodinid butterflies were found only in Krishnopur; likewise acraeid butterflies were found only in AERE campus (Table 2).

Table 1 Butterfly species recorded from 4 different areas of Savar, Bangladesh.

\begin{tabular}{|c|c|c|c|}
\hline Common names & Families and scientific Names & Status & Distribution \\
\hline \multicolumn{4}{|c|}{ Family- Papilionidae } \\
\hline 1. Common Mime & Chilasa clytia clytia & NR & $\mathrm{K}, \mathrm{A}, \mathrm{H}, \mathrm{R}$ \\
\hline 2. Lime Swallowtail & Papilio demoleus demoleus & VC & $\mathrm{K}, \mathrm{A}, \mathrm{H}, \mathrm{R}$ \\
\hline 3. White head Batwing & Papilio sycorax & NR & $\mathrm{K}, \mathrm{A}, \mathrm{H}, \mathrm{R}$ \\
\hline 4. Red Helen & Papilio helenus helenus & VR & $\mathrm{K}$ \\
\hline 5. Common Mormon & Papilio polytes polytes & $\mathrm{C}$ & $\mathrm{K}, \mathrm{A}, \mathrm{H}, \mathrm{R}$ \\
\hline $6 .^{*}$ & *Papilio polytes romulus & NR & $\mathrm{K}$ \\
\hline 7. Great Mormon & Papilio memnon agenor & VR & $\mathrm{K}$ \\
\hline 8. * & * Papilio sp. & VR & K \\
\hline 9. * & *Papilio sp. & VR & $\mathrm{K}$ \\
\hline 10. *Common Rose & ${ }^{\star}$ Pachliopta aristrochiae goniopeltis & VR & $\mathrm{A}, \mathrm{H}$ \\
\hline 11 * & ${ }^{\star}$ Pachliopta aristrochiae asteris & NR & $\mathrm{K}$ \\
\hline 12. *Common Jay & *Graphium doson axion & $\mathrm{R}$ & A \\
\hline 13. Tailed Jay & Graphium agamemnon agamemnon & NR & $\mathrm{K}, \mathrm{A}$ \\
\hline 14. *Great Jay & *Graphium eurypylus cheronus & VR & A \\
\hline \multicolumn{4}{|c|}{ Family- Danaidae } \\
\hline 1. Plane Tiger & Danaus chrysippus chrysippus & VC & $\mathrm{K}, \mathrm{A}, \mathrm{H}, \mathrm{R}$ \\
\hline $\begin{array}{l}\text { 2. Common Tiger/ } \\
\text { Indian Monarch }\end{array}$ & Danaus genutia genutia & VC & $\mathrm{K}, \mathrm{A}, \mathrm{H}, \mathrm{R}$ \\
\hline 3. Blue Tiger & Tirumala limniace limniace & C & $\mathrm{K}, \mathrm{A}, \mathrm{H} . \mathrm{R}$ \\
\hline 4. Common Indian Crow & Euploea core godartii & VC & $\mathrm{K}, \mathrm{A}, \mathrm{H} . \mathrm{R}$ \\
\hline 5. *Striped Blue Crow & ${ }^{\star}$ Euploea mulciber mulciber & VR & $\mathrm{A}$ \\
\hline
\end{tabular}




\begin{tabular}{|c|c|c|c|}
\hline Common names & Families and scientific Names & Status & Distribution \\
\hline \multicolumn{4}{|c|}{ Family- Pieridae } \\
\hline 1. Red-Spot Jezebel & Delias descombesi descombesi & $\mathrm{R}$ & $\mathrm{H}$ \\
\hline 2. Painted Jezebel & Delias hyparete indica & VC & $\mathrm{K}, \mathrm{A}, \mathrm{H} . \mathrm{R}$ \\
\hline 3. Common Gull & Cepora nerissa dapha & $\mathrm{C}$ & $A, H . R$ \\
\hline 4. Striped Albatross & Appias libythea olferna & VC & A,H.R \\
\hline 5. ${ }^{\star}$ Ceylon Lesser Albatross & ${ }^{*}$ Appias paulina adamsoni & NR & A \\
\hline 6.Great Orange Tip & Hebomoia glaucippe glaucippe & $\mathrm{R}$ & $A, R$ \\
\hline 7. Pale Wanderer & Pareronia anais & VC & $\mathrm{K}, \mathrm{A}, \mathrm{H} . \mathrm{R}$ \\
\hline 8. Mottled Emigrant & Catopsilia pyranthe pyranthe & VC & $\mathrm{K}, \mathrm{A}, \mathrm{H}, \mathrm{R}$ \\
\hline 9. Lemon Emigrant & Catopsilia pomona pomona & VC & $\mathrm{K}, \mathrm{A}, \mathrm{H}, \mathrm{R}$ \\
\hline 10.*Common Emigrant & ${ }^{*}$ Catopsilia pomona jugurtha & $\mathrm{R}$ & A. \\
\hline 11.* & ${ }^{\star}$ Catopsilia pomona hilaria & VR & $\mathrm{K}, \mathrm{A} . \mathrm{R}$ \\
\hline 12.* & ${ }^{*}$ Catopsilia pomona catilia & $\mathrm{R}$ & K,A.R \\
\hline 13.* & ${ }^{*}$ Catopsilia pomona alcmeone & VR & $\mathrm{K}, \mathrm{A}, \mathrm{H}$ \\
\hline 14. *Psyche & Leptosia nina nina & VC & $\mathrm{K}, \mathrm{A}, \mathrm{H}, \mathrm{R}$ \\
\hline 15. & ${ }^{\star}$ Artogeia canidia indica & VR & A \\
\hline 16.*Yellow Orange Tip & */xias pyrene verna & VR & $\mathrm{K}$ \\
\hline $17 .^{*}$ & ${ }^{*}$ Saletara liberia distanti & VR & $A$ \\
\hline 18. Common Grass Yellow & Eurema hecabe contubernalis & VC & $\mathrm{K}, \mathrm{A}, \mathrm{H}, \mathrm{R}$ \\
\hline 19*One-Spot Grass Yellow & ${ }^{\star}$ Eurema andersonii andersonii & NR & A \\
\hline $20 *^{*}$ & ${ }^{\star}$ Eurema ada jona & NR & A \\
\hline 21. & Eurema simulatrix tecmessa & NR & $A$ \\
\hline 22.* & ${ }^{\star}$ Eurema simulatrix inouei & VC & $\mathrm{K}, \mathrm{A}$ \\
\hline \multicolumn{4}{|c|}{ Family- Satyridae } \\
\hline 1.Common Evening Brown & Melanitis leda leda & VC & $\mathrm{K}, \mathrm{A}, \mathrm{H}, \mathrm{R}$ \\
\hline 2. Common Palmfly & Elymnias hypermnestra tinctoria & VC & $\mathrm{K}, \mathrm{A}, \mathrm{H}, \mathrm{R}$ \\
\hline 3.* & ${ }^{*}$ Elymnias hypermnestra meridionalis & VC & $\mathrm{K}$ \\
\hline 4.Dark-Brand Bushbrown & Mycalesis mineus mineus & VC & $\mathrm{K}, \mathrm{A}, \mathrm{H}, \mathrm{R}$ \\
\hline 5. Lilacine Bushbrown & Mycalesis francisca sanatana & VC & $\mathrm{K}$ \\
\hline 6. Common Bushbrown & Mycalesis perseus cepheus & NR & $\mathrm{K}$ \\
\hline 7.*Burmese Bushbrown & ${ }^{\star}$ Mycalesis perseoides perseoides & VR & $\mathrm{K}$ \\
\hline 8.* & ${ }^{*}$ Mycalesis intermedia & VR & $\mathrm{K}$ \\
\hline 9.* & ${ }^{*}$ Mycalesis sangaica tunicula & VR & $\mathrm{K}$ \\
\hline 10.*Nigger & *Orsotriaena medus medus & $\mathrm{R}$ & $\mathrm{K}$ \\
\hline 11. *Bamboo Treebrown & *Lethe europa niladana & $\mathrm{R}$ & $\mathrm{K}$ \\
\hline 12. ${ }^{\star}$ Common Fivering & *Ypthima baldus baldus & $\mathrm{R}$ & $\mathrm{K}$ \\
\hline 13.* & ${ }^{*}$ Ypthima nebulosa & VR & $\mathrm{K}$ \\
\hline 14. Common Fourring & Ypthima huebneri & $\mathrm{R}$ & $\mathrm{K}$ \\
\hline \multicolumn{4}{|c|}{ Family- Amathusiidae } \\
\hline 1. *Duffer & Dischophora sondaica zal & NR & $\mathrm{K}$ \\
\hline \multicolumn{4}{|c|}{ Family- Riodinidae } \\
\hline 1. Palm Judy & Abisara echerius paionea & VR & $\mathrm{K}$ \\
\hline 2. Punchinellow & Zemeros flegyas flegyas & VR & $\mathrm{K}$ \\
\hline 3. & ${ }^{\star}$ Abisara abnormis & VR & $\mathrm{K}$ \\
\hline \multicolumn{4}{|c|}{ Family- Acraeidae } \\
\hline 1.Tawny Coster & Acraea violae & VC & $A$ \\
\hline \multicolumn{4}{|c|}{ Family- Nymphalidae } \\
\hline 1. Leopard Lacewing & Cethosia cynae euanthes & C & $\mathrm{K}, \mathrm{A}, \mathrm{H}, \mathrm{R}$ \\
\hline 2. Common Leopard & Phalanta phalantha phalantha & VC & $\mathrm{K}, \mathrm{A}, \mathrm{H}, \mathrm{R}$ \\
\hline 3. Chocolate Pansy & Junonia iphita iphita & $\mathrm{R}$ & $\mathrm{K}, \mathrm{A}$ \\
\hline 4. Grey Pansy & Junonia atlites atlites & VC & $\mathrm{K}, \mathrm{A}, \mathrm{H}, \mathrm{R}$ \\
\hline 5. Peacock Pansy & Junonia almana almana & VC & $\mathrm{K}, \mathrm{A}, \mathrm{H}, \mathrm{R}$ \\
\hline 6. Lemon Pansy & Junonia lemonias lemonias & VC & $\mathrm{K}, \mathrm{A}, \mathrm{H}, \mathrm{R}$ \\
\hline 7. Yellow Pansy & Junonia hierta hierta & $\mathrm{C}$ & $\mathrm{K}, \mathrm{H}$ \\
\hline 8. *Blue Pansy & *Junonia orithya ocyale & VR & $\mathrm{K}$ \\
\hline 9. Great Eggfly & Hypolimnas bolina jacintha & $\mathrm{C}$ & $\mathrm{K}, \mathrm{A}, \mathrm{H}, \mathrm{R}$ \\
\hline 10. Common Castor & Ariadne merione tapestrina & VC & $\mathrm{K}, \mathrm{R}$ \\
\hline 11. Common Baron & Euthalia aconthea apana & $\mathrm{C}$ & $\mathrm{K}, \mathrm{A}, \mathrm{H}, \mathrm{R}$ \\
\hline 12. & ${ }^{\star}$ Euthalia alpheda verena & VR & K.A \\
\hline
\end{tabular}




\begin{tabular}{|c|c|c|c|}
\hline Common names & Families and scientific Names & Status & Distribution \\
\hline 13. ${ }^{\star}$ Common Sergent & ${ }^{*}$ Athyma perius perius & VR & $A$ \\
\hline 14. Himalayan Sergent & Athyma opalina shan & $\mathrm{C}$ & $\mathrm{K}, \mathrm{A}, \mathrm{H}$ \\
\hline 15. Color Sergent & Athyma inara & $\mathrm{R}$ & $\mathrm{H}$ \\
\hline 16. ${ }^{\star}$ Common Sailor & ${ }^{\star}$ Neptis hylas & $\mathrm{R}$ & $\mathrm{H}$ \\
\hline 17.Chestnut-Streaked Sailer & Neptis jumbah jumbah & VC & $\mathrm{H}$ \\
\hline 18. *Commander & ${ }^{\star}$ Moduza procris procris & VR & $A$ \\
\hline 19.*Tawny Rajah & ${ }^{\star}$ Charaxes bernardus hierax & VR & $\mathrm{K}$ \\
\hline \multicolumn{4}{|c|}{ Family- Lycaenidae } \\
\hline 1. & Allotinus unicolor unicolor & $\mathrm{R}$ & A \\
\hline 2 & Celastrina transpecta & NR & $\mathrm{K}, \mathrm{A}$ \\
\hline 3. Common Pierrot & Castalius rosimon rosimon & VC & $\mathrm{K}, \mathrm{A}, \mathrm{H}, \mathrm{R}$ \\
\hline 4. Spotted Pierrot & Tarucus callinara & $\mathrm{R}$ & $\mathrm{K}$ \\
\hline 5. Angle Pierrot & Caleta caleta decidia & NR & $\mathrm{A}, \mathrm{H}$ \\
\hline 6. Lime Blue & Chylades lajus lajus & $\mathrm{C}$ & $A$ \\
\hline 7.* & *Chylades lajus tavoyanus & C & $A$ \\
\hline 8. Plains Cupid & Chylades pandava pandava & C & A \\
\hline 9. Forest Quaker & Zizula hylax hylax & $\mathrm{R}$ & $\mathrm{K}, \mathrm{R}$ \\
\hline 10. Opaque Six-Lineblue & Nacaduba beroe gythion & $\mathrm{R}$ & $\mathrm{K}$ \\
\hline 11.* & ${ }^{\star}$ Catochrysops panormus exguus & $\mathrm{C}$ & $A$ \\
\hline 12. Common Cerulean & Jamides celeno celeno & $\mathrm{R}$ & $\mathrm{K}, \mathrm{A}$ \\
\hline 13.*Dark Cerulean & *Jamides bochus bochus & $\mathrm{R}$ & $\mathrm{K}, \mathrm{A}$ \\
\hline 14. *Pea Blue & *Lampides boeticus boeticus & VR & $A$ \\
\hline 15. ${ }^{*}$ Common Red Flash & ${ }^{\star}$ Rapala airbus iarbus & $\mathrm{R}$ & K \\
\hline 16. Slat Flash & Rapala manea schistacea & C & $\mathrm{K}, \mathrm{A}$ \\
\hline 17. ${ }^{\star}$ Scarce Slat Flash & ${ }^{*}$ Rapala scintilla scitilla & VR & $\mathrm{K}$ \\
\hline 18. *Scarlet Flash & ${ }^{*}$ Rapala diences diences & NR & $\mathrm{K}$ \\
\hline 19. Cornelian & Deudorix epijarbas amatius & VR & $\mathrm{K}$ \\
\hline 20. Chocolate Royal & Remelana jangala ravata & $\mathrm{C}$ & $\mathrm{K}$ \\
\hline 21. Long-Banded Silverline & Spindasis lohita batina & $\mathrm{R}$ & $\mathrm{K}$ \\
\hline 22.*Club Silverline & *Spindasis syama latipicta & $\mathrm{R}$ & $\mathrm{K}$ \\
\hline 23. & Acytolepis lenya & $\mathrm{C}$ & $\mathrm{K}, \mathrm{A}$ \\
\hline 24. *Gram Blue & ${ }^{\star}$ Euchrysops cnejus cnejus & VR & $\mathrm{K}$ \\
\hline $25 . *$ & *Miletus mallas mallas & VR & $A$ \\
\hline $26 .{ }^{*}$ & *Miletus nymphis fictus & VR & $A$ \\
\hline 27. Yamfly & Loxura atymnus continentalis & VC & $\mathrm{K}, \mathrm{A}, \mathrm{H}$ \\
\hline 28. *Quaker & *Neopithecops zalmora zalmora & $\mathrm{R}$ & $\mathrm{K}$ \\
\hline 29.*Apely & *Spalgis epius epius & $\mathrm{R}$ & $\mathrm{K}, \mathrm{R}$ \\
\hline 30.* & *Spalgis sp. & C & $\mathrm{K}$ \\
\hline 31.* & ${ }^{\star}$ Callenya melaena & VR & K \\
\hline 32.*Zebra-Blue & *Syntarucus plinius & VR & $\mathrm{K}$ \\
\hline 33. ${ }^{*}$ Tailless Line Blue & ${ }^{*}$ Prosotas dubiosa indica & VR & $\mathrm{K}, \mathrm{R}$ \\
\hline 34. ${ }^{*}$ Common Line Blue & ${ }^{\star}$ Prosotas nora ardates & $\mathrm{R}$ & $\mathrm{K}, \mathrm{R}$ \\
\hline 35. ${ }^{*}$ ale Grass Blue & *Zizeeria maha maha & VR & $\mathrm{K}$ \\
\hline 36. ${ }^{\star}$ Dark Grass Blue & *Zizeeria karsandra karsandra & NR & $K, R$ \\
\hline 37.*Lesser Grass Blue & *Zizina otis sangra & VR & $\mathrm{K}$ \\
\hline 38. ${ }^{*}$ Pointed Ciliate Blue & ${ }^{*}$ Anthene lycaenina lycambes & VR & $\mathrm{R}$ \\
\hline 39. ${ }^{*}$ Ciliate Blue & ${ }^{*}$ Anthene emolus emolus & VR & $\mathrm{K}$ \\
\hline 40. ${ }^{*}$ Common Acacia Blue & *Surendra quercetorum quercetorum & NR & $A$ \\
\hline 41. *Peacock Royal & *Tajuria cippus maxentius & VR & $\mathrm{H}$ \\
\hline 42. *Sunbeam & ${ }^{\star}$ Curetis thetis & VR & $\mathrm{K}$ \\
\hline 43. *Sunbeam & ${ }^{\star}$ Curetus sp. & $\mathrm{R}$ & $\mathrm{K}$ \\
\hline 44.Common Oakblue & Arhopala pseudocentaurus nakula & $\mathrm{C}$ & $\mathrm{K}, \mathrm{H}$ \\
\hline 45.*Indian Cupid & ${ }^{\star}$ Everes lacturnus lacturnus & VR & $\mathrm{K}$ \\
\hline 46.* & ${ }^{*}$ Aphnaeus ictis & VR & A \\
\hline 47.* & *Drupadia sp. & VR & $\mathrm{K}, \mathrm{R}$ \\
\hline
\end{tabular}




\begin{tabular}{|c|c|c|c|}
\hline Common names & Families and scientific Names & Status & Distribution \\
\hline \multicolumn{4}{|c|}{ Family- Hesperiidae } \\
\hline 1.*Palm Redeye & ${ }^{\star}$ Erionata torus & $\mathrm{R}$ & $A$ \\
\hline 2. Giant Redeye & Gangara thyrsis thyrsis & VR & $\mathrm{K}, \mathrm{A}$ \\
\hline 3. Brown Redeye & Badamia exclamationis & $\mathrm{R}$ & $\mathrm{K}, \mathrm{A}$ \\
\hline 4. Grass Demon & Udaspes folus & NR & $\mathrm{K}, \mathrm{H}$ \\
\hline 5. Common Snow Flat & Tagiades japetus ravi & VC & $\mathrm{K}$ \\
\hline 6. *Snow Flat & *Tagiades japetus atticus & $\mathrm{R}$ & $\mathrm{K}$ \\
\hline $7 .^{*}$ & ${ }^{\star}$ Ancistroides nigrita diocles & $\mathrm{R}$ & $A$ \\
\hline 8. ${ }^{\star}$ Chestnut Angle & ${ }^{*}$ Odontoptilum angulatum angulatum & NR & $\mathrm{K}$ \\
\hline $9 .{ }^{*}$ & ${ }^{*}$ Caltoris brunnea caere & VR & $\mathrm{K}$ \\
\hline 10.*Blank Swift & ${ }^{\star}$ Caltoris kumara moorei & VR & $\mathrm{K}$ \\
\hline 11. ${ }^{*}$ Common Banded Demon & *Notocrypta paralysos asawa & VR & $\mathrm{K}$ \\
\hline 12.*Tree Filtter & ${ }^{\star}$ Hyarotis adrastus praba & VR & $\mathrm{H}$ \\
\hline 13. Grey Brand-Redeye & Matapa druna & $\mathrm{C}$ & $K, A$ \\
\hline 14. ${ }^{\star}$ Common Redeye & *Matapa aria & VR & $\mathrm{K}$ \\
\hline 15. Contiguous swift & Polytremis lubricans lubricans & NR & $\mathrm{K}, \mathrm{A}, \mathrm{H}, \mathrm{R}$ \\
\hline 16.Yellow Spot Swift & Polytremis eltola eltola & VC & $\mathrm{K}, \mathrm{A}, \mathrm{H}, \mathrm{R}$ \\
\hline 17. ${ }^{\star}$ Indian Palm Bob & *Suastus gremius gremius & VR & $\mathrm{K}$ \\
\hline 18. ${ }^{\star S}$ mall Branded Swift & ${ }^{\star}$ Pelopidas mathius mathius & $\mathrm{R}$ & $\mathrm{K}$ \\
\hline 19. *Great Swift & ${ }^{*}$ Pelopidas assamensis & VR & A \\
\hline $20 .^{*}$ & ${ }^{\star}$ Pelopidas agna agna & VR & $\mathrm{K}, \mathrm{A}$ \\
\hline $21 .^{*}$ & ${ }^{\star}$ Halpe porus & VC & $\mathrm{K}$ \\
\hline 22. ${ }^{\star}$ Straight Swift & *Parnara naso bada & VR & $\mathrm{K}, \mathrm{R}$ \\
\hline 23.Straight Swift & Parnara guttata apostate & VC & $\mathrm{K}, \mathrm{A}, \mathrm{H}, \mathrm{R}$ \\
\hline 24.Chestnut Bob & lambrix salsala salsala & $\mathrm{C}$ & $\mathrm{K}, \mathrm{A}$ \\
\hline $25 .^{*}$ & *lambrix sp. & NR & $\mathrm{K}$ \\
\hline 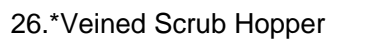 & ${ }^{\star}$ Aeromachus stigmatus shanda & $\mathrm{R}$ & $\mathrm{K}$ \\
\hline 27.Pigmy Scrub Hopper & Aeromachus pygmaeus & VR & $\mathrm{K}$ \\
\hline $28 .^{*}$ & ${ }^{*}$ Telicota hilda & $\mathrm{R}$ & $\mathrm{K}$ \\
\hline $29 .^{*}$ & *Telicota besta besta & VR & K \\
\hline 30.*Pale Palm Dart & ${ }^{\star}$ Telicota colon stinga & VR & $\mathrm{K}$ \\
\hline 31.* & ${ }^{\star}$ Telicota linna linna & VR & $\mathrm{K}$ \\
\hline 32. ${ }^{*}$ Common Dartlet & *Oriens gola pseudolus & NR & $\mathrm{K}$ \\
\hline
\end{tabular}

$\mathrm{K}=$ Krishnopur, $\mathrm{A}=\mathrm{AERE}$ Campus, $\mathrm{H}=$ Horters (part of JU Campus) and R=Rajalak Farm; * Newly- recorded species for Bangladesh

Table 2 Butterfly species diversity recorded from the study areas at Savar, Bangladesh

\begin{tabular}{lcccc}
\hline \multirow{2}{*}{ Families } & \multicolumn{5}{c}{ Butterflies collected from the study areas } \\
\cline { 2 - 5 } & Krishnopur & $\begin{array}{c}\text { AERE } \\
\text { Campus }\end{array}$ & Horters & Rajalak Farm \\
\hline Papilionidae & 12 & 9 & 8 & 7 \\
Danaidae & 4 & 5 & 4 & 4 \\
Pieridae & 17 & 18 & 13 & 11 \\
Satyridae & 13 & 6 & 5 & 5 \\
Amathusiidae & 1 & 0 & 0 & 0 \\
Riodinidae & 3 & 0 & 0 & 0 \\
Acraeidae & 0 & 1 & 0 & 0 \\
Nymphalidae & 16 & 16 & 14 & 12 \\
Lycaenidae & 33 & 22 & 17 & 17 \\
Hesperiidae & 31 & 10 & 11 & 9 \\
\hline Total & $\mathbf{1 3 0}$ & $\mathbf{8 7}$ & $\mathbf{7 2}$ & $\mathbf{6 5}$ \\
\hline
\end{tabular}

Nineteen of Nymphalidae (6 newly recorded species), 14 of Satyridae (8 new), 14 of Papilionidae (7 new), and 5 of Danaidae (1 new) butterflies were identified. In addition, a single species of Acraeidae, 3 of Riodinidae and a single species each of Amathusiidae, Riodinidae and Amathusiidae butterflies were recorded to be new for the country (Table 1).

All 158 species of butterflies were identified and classified into VC, C, NR, R and VR categories which are presented in Table 3. More than half of butterfly species were judged to be classified into VR or R categories, the species numbers of which reached 56 and 32 (35.44\% and 20.25\%), respectively, whereas those of the other 30,19 and 21 species were classified into VC, C and NR categories, the proportions of which were $12.18 \%$, $12.03 \%$ and $13.29 \%$, respectively. 
Table 3 Classification of butterfly species according to their availability at selected study areas at Savar.

\begin{tabular}{|c|c|c|c|c|c|c|c|c|c|c|}
\hline \multirow{3}{*}{ Families } & \multicolumn{10}{|c|}{ Number and percentages of variously categorized butterfly species } \\
\hline & \multicolumn{2}{|c|}{ VC } & \multicolumn{2}{|c|}{$\mathrm{C}$} & \multicolumn{2}{|c|}{ NR } & \multicolumn{2}{|c|}{$\mathrm{R}$} & \multicolumn{2}{|c|}{ VR } \\
\hline & Total & $\%$ & Total & $\%$ & Total & $\%$ & Total & $\%$ & Total & $\%$ \\
\hline Papilionidae & 1 & 7.14 & 1 & 7.14 & 5 & 35.71 & 1 & 7.14 & 6 & 42.86 \\
\hline Danaidae & 3 & 60.00 & 1 & 20.00 & 0 & 00.00 & 0 & 0.00 & 1 & 20.00 \\
\hline Pieridae & 8 & 36.37 & 1 & 4.55 & 4 & 18.18 & 4 & 18.18 & 5 & 22.27 \\
\hline Satyridae & 5 & 35.71 & 0 & 0.00 & 1 & 7.14 & 4 & 28.57 & 4 & 28.57 \\
\hline Amathusiidae & 0 & 0.00 & 0 & 0.00 & 1 & 100.00 & 0 & 00.00 & 0 & 0.00 \\
\hline Riodinidae & 0 & 0.00 & 0 & 0.00 & 0 & 0.00 & 0 & 0.00 & 3 & 100.00 \\
\hline Acraeidae & 1 & 100.00 & 0 & 0.00 & 0 & 0.00 & 0 & 0.00 & 0 & 0.00 \\
\hline Nymphalidae & 6 & 31.58 & 5 & 26.32 & 0 & 0.00 & 3 & 15.79 & 5 & 26.32 \\
\hline Lycaenidae & 2 & 4.24 & 9 & 19.15 & 5 & 10.64 & 13 & 27.66 & 18 & 38.30 \\
\hline Hesperiidae & 4 & 12.50 & 2 & 6.25 & 5 & 15.63 & 7 & 21.88 & 14 & 43.57 \\
\hline Total & 30 & 18.99 & 19 & 12.03 & 21 & 13.29 & 32 & 20.25 & 56 & 35.44 \\
\hline
\end{tabular}

VC=Very Common (>100 species), C=Common (50-100 species), NR=Not Rare (15-50 species), R=Rare (2-15 species) and VR=Very Rare $<2$ species).

Relatively large proportions of VR and R species belonged to Lycaenidae (18 and 13 species) and Hesperiidae (14 and 7 species). The numbers of Lycaenidae and Hesperiidae species were found to show larger fluctuations among the four study areas as compared to those of the other families (Table 3).

Here we identified 158 species of butterflies, 87 species of which were newly-recorded in Bangladesh and rest 71 species were reported earlier (Islam et al., 2007). The VR and R categories were respectively 56 and 32 (35.44\% and $20.25 \%$ ) of 158 butterfly species (Tables 1 and 3). Numbers of Papilionidae and Danaidae species of butterflies, which are known to show long-distance flights, were identified in all study areas, whereas large numbers of species of Lycaenidae and Hesperidae, which show shortdistance flights, were identified in only Krishnopur and AERE Campus. In addition to the differences in floral types in and around the study areas, perhaps the flying ability of the butterflies is a significant factor to determine the species diversity. That is, larvae of many butterfly species feed on leaves of specific or relatively limited plants. The types of floral abundance in the study areas seem to vary depending on such vegetative conditions as farms, gardens and forests, essential for serving the foods for butterflies (Vickery, 1998).
Although the present 87 species of butterflies were newly recorded in Bangladesh, we did not find 9 species which were identified in our previous studies (Islam et al., 2007). These were Papilio helenus helenus, Papilio memnon agenor (Papilionidae), Ypthima hubneri (Satyridae), Abisara echerius paionea, Zemeros flegyas flegyas (Riodinidae), Junonia iphita iphita, Athyma inara (Nymphalidae), Deudorix epijarbas amatius (Lycaenidae) and Aeromachus pygmaeus (Hesperiidae). This does not suggest only a possibility that we failed to find these species in our present study, but there is also another possibility that Savar and its surrounding areas lost the ability to support the populations of those butterfly species due to the damage of vegetations by industrial and residential activities like gardening, frequent grass-cuttings and cultivation for vegetables, fruits and flowers (Dennis \& Williams, 1986). In contrast, 30 and 19 species of butterflies were classified into VC and C categories, respectively, which seemed to adapt the disturbances of ecological conditions owing to the industrial and residential activities mentioned above.

The maximum 130 species of butterflies were identified in Krishnopur, where the species diversity of butterflies may be supported by various types of environmental and vegetative conditions such as standing forests, grasslands and cultivating lands for agriculture. The minimum 
number of butterfly species (65 species) was recorded in Rajalak Farm, the environmental and vegetative conditions of which may not be congenial for supporting the diversity of butterfly species. The diversity of butterfly species or other organisms is supposed to depend on diversities of environmental and vegetative conditions, the damage of which seem to be accompanied by the contraction of species diversity. The present results may be useful to estimate the actual and transitional state of environmental and vegetative conditions in and around the study areas and to search a policy for conserving our natural resources on the development of industrial and residential activities.

\section{Acknowledgements}

This work was conducted under IFRB (Bangladesh) and YU (Japan) agreement for research cooperation (2010-2015). ATMF Islam and Akira Yamanaka were the respective national counterparts.

\section{References}

Dennis, R.L.H.\& Williams, W.R. 1986. Mate locating behaviour of the large skipper butterflyOchlodes venata: flexible strategies and spatial components. J. Lepid. Soc. 41: $45-64$.

Islam, A.T.M.F., Razzak, M.A., Mamun, A.N.K., Saifullah, A.S.M., Shahjahan, R.M., Endo, K., Yamanaka, A., Kometani, M. \& Inoue, M. 2007. Common Butterflies in Bangladesh, 33p (with 15 plates), Published by Institute of Food \& Radiation Biology, AERE, Savar, Dhaka.

Kurani, E.J. \& Pinratana, A. 1981. Butterflies in Thailand. Vol. 4. The Viratham Press, Bangkok. pp. 215
Kurani, E.J. \& Pinratana, A. 1983. Butterflies in Thailand. Vol. 2. revised ed, The Viratham Press, Bangkok. pp. 71.

Kurani, E.J. \& Pinratana, A. 1985. Butterflies in Thailand. Vol. 5. The Viratham Press, Bangkok. pp.152.

Pinratana, A. \& Eliot, J. N. 1982. Butterflies in Thailand. Vol. 1. 2nd and revised ed. Bosco Offset, Bangkok. pp.78.

Pinratana, A. \& Eliot, J. N. 1996a. Butterflies in Thailand. Vol. 6. The Viratham Press, Bangkok. pp.140.

Pinratana, A. \& Eliot, J. N. 1996b. Butterflies in Thailand. Vol. 3. 2nd and revised ed. Bosco Offset, Bangkok. pp.140.

Robbins, R.K. \& Opler, P.A. 1997. Butterfly diversity and a preliminary comparison with bird and mammal diversity. In: Biodiversity II, understanding and protecting our biological resources, Wilson, D. E., M.L. Reaka-Kudla and E.O. Wilson (Eds.). Joseph Henry Press, Washington, DC.

Shahjahan, R.M., Abdur Razzak, M., Fayezul, A.T.M.F., Saifullah, A.S.M., Yamanaka. Inoue, M. \& Endo, K. 2008. Field studies of butterflies: A trial for the establishment of indices to evaluate the seasonal fluctuation and the ecological states of forests in Bangladesh. Univ. j. zool. Rajshahi Univ. 27: 67-71.

Tiple, A.D., Deshmukh, V.P. \& Dennis, R.L.H. 2006. Factors influencing nectar plant resource visits by butterflies on a university campus: implications for conservation. Nota Lepid. 28: 213-224.

Tiple, A.D., Khurad, A.M. \& Dennis, R.L.H. 2007. Butterfly diversity in relation to a human-impact gradient on an Indian university campus. Nota Lepid. 30(1): 179-188.

Vickery, M. 1998. Gardening of butterflies. The British Butterflies Conservation Society, Dedham. 44 pp.

Wynther-Blyth, M.A. 1957. Butterflies of the Indian Region. Bombay Natural History Society. 